\title{
Positron study of carbon black parameters: Structure and Surface Area
}

\author{
J. Wang ${ }^{1}$, V. O. Jobando ${ }^{1,2}$, and C. A. Quarles ${ }^{1 *}$ \\ ${ }^{1}$ Department of Physics and Astronomy, Texas Christian University, TCU Box 298840, \\ Fort Worth TX 76129 USA \\ ${ }^{2}$ Spokane Community College, Spokane WA USA \\ *Corresponding author, email address: c.quarles@tcu.edu
}

Keywords: Carbon black, Positron lifetime, N2SA, CDBP

\begin{abstract}
Carbon black (CB) is essentially carbon in the form of extremely fine particle aggregates (100-200nm) having an amorphous molecular structure. Oil absorption or compressed dibutyl phthalate (CDBP) and iodine adsorption or nitrogen surface area (N2SA) are parameters commonly used to describe $\mathrm{CB}$ morphology or structure and the surface area of $\mathrm{CB}$ available for reaction with rubber. We have used both positron lifetime and Doppler broadening spectroscopy to study these two parameters for a variety of rubber CB composite samples. The o-Ps pickoff lifetime depends only on the type of rubber and is independent of CDBP and N2SA. However, the o-Ps lifetime intensity, I3, and the S parameter decrease with both CDBP and N2SA.
\end{abstract}

\section{Introduction}

Carbon black (CB) [2] is an industrial product. The main effect of adding $\mathrm{CB}$ into a rubber is to strengthen the rubber. Compressed dibutyl phthalate (CDBP) and nitrogen surface area (N2SA) are two parameters that describe $\mathrm{CB}$ morphology and surface area of $\mathrm{CB}$ exposed for reaction with rubber $[3,4]$. The purpose of this research is to investigate the dependence of the positron annihilation lifetime spectroscopy (PALS) and Doppler broadening spectroscopy (DBS) on these two CB parameters for a variety of rubber CB composite samples.

\section{Experimental details}

The lifetime apparatus was a typical fast-fast coincidence system using $\mathrm{BaF}_{2}$ scintillators, Photonis phototubes and Ortec 583 Constant Fraction Differential Discriminators. The data were collected with an Ortec Trump MCA in a PC computer. The timing resolution was about 350 ps. Two samples were sandwiched around a ${ }^{22} N a_{11}$ source, $50 \mu \mathrm{Ci}$ deposited on $0.00125 \mathrm{~cm}$ nickel foil. No source correction was made since the source did not exhibit any long lifetime component, all the samples had the same average atomic number and thus the same source correction and we were only interested in relative values of the o-Ps pickoff intensity.

The Sid Richardson Carbon Co., Fort Worth, TX, provided the samples. For pure CB samples, the $\mathrm{CB}$ powder was pressed into a cylindrical aluminum sample holder of $50 \mathrm{~mm}$ diameter and $20 \mathrm{~mm}$ thickness. For composite samples the rubber, $\mathrm{CB}$ and vulcanizing additives were mixed according to ASTM D3191 and vulcanized in a mold to produce cylindrical samples of $38 \mathrm{~mm}$ diameter and $10 \mathrm{~mm}$ thick. All samples had a concentration of $50 \mathrm{~g}$ of CB to $100 \mathrm{~g}$ of rubber ( $50 \mathrm{phr}$ ). The rubber component included natural rubber, and two solution styrene butadiene rubbers, Sn-SSBR and D706 rubber. A variety of CB types were selected to have a range of CDBP and N2SA. CB types are all the same composition, but differ in morphology. The CBs with lower number are more 
branched while those of higher numbers are more spheroidal in structure. The CBs investigated included N110, N220, N326, N330, N339, N347, N358, N650, N660 and N772. (For more details: http://www.sidrich.com/Products/Tire.htm)

\section{Results and discussion}

The lifetime spectra were analyzed into three components using the LT program.[1] The shortest lifetime was fixed to 125 ps to stabilize the non-linear fit. The intermediate lifetime, Tau2, was attributed to annihilation in the bulk of both the rubber and the CB. The long lifetime component, Tau3, was attributed to ortho-positronium (o-Ps) pickoff.

In Fig. 1, the o-Ps pickoff lifetime, Tau3, is plotted versus $\mathrm{CDBP}$ of the $\mathrm{CB}$ in the samples respectively. A similar result is found for Tau3 vs N2SA. Tau3 is independent of both CDBP and N2SA; but it depends

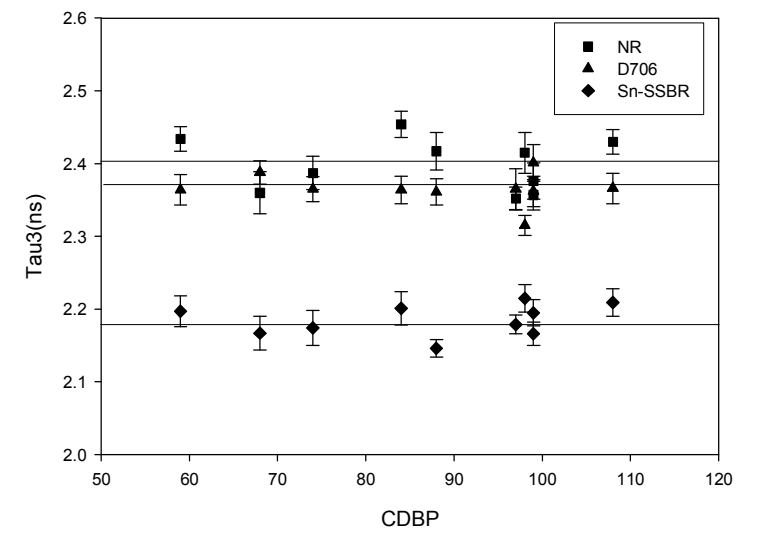

Fig. 1 The O-Ps pickoff lifetime vs CDBP on the type of rubber. In the pure CB samples, there was less than $2 \%$ intensity for a long lifetime component, which was due to annihilation in the thin plastic covers and absorbed water in the $\mathrm{CB}$ and not to positronium formation in the $\mathrm{CB}$ itself. Thus in the composite samples, we attribute the o-Ps pickoff to the rubber and not to the $\mathrm{CB}$.

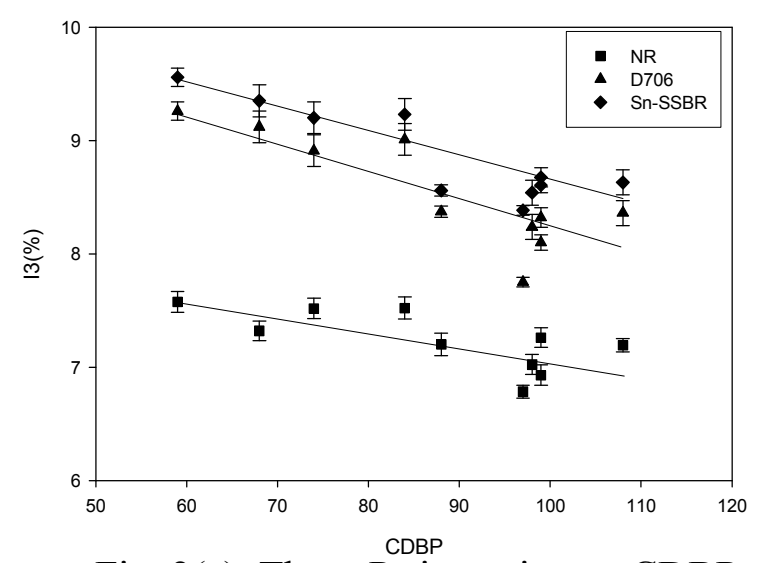

Fig. 2(a) The o-Ps intensity vs. CDBP



Fig. 2(b) The o-Ps intensity vs. N2SA

In Figs. 2(a) and (b), the o-Ps pickoff lifetime intensity, I3, is plotted versus CDBP and N2SA respectively. I3 decreases with both CDBP and N2SA. Since there is no significant positronium formation in the $\mathrm{CB}$ material, the effect of adding $\mathrm{CB}$ to the composite is mainly to reduce the number of positrons available to form positronium in the rubber. A CB with a larger surface area (higher N2SA) or a more open, flat structure (higher CDBP) provides higher probability for the positron to stop in the $\mathrm{CB}$ particles so less positronium is formed in the rubber.

In Fig. 3, the $\mathrm{S}$ parameter is shown versus N2SA of the CB respectively. A similar result is found for $\mathrm{S}$ vs CDBP. Like I3, S decreases with both CDBP and N2SA. This is due mainly to the 
fraction of positrons annihilating in the CB particles in the composite and therefore not being available to form positronium in the rubber. However, unlike I3, S is independent of the type of rubber. The three separated trend lines shown in Fig. 2(a) and (b), essentially collapse into one shown in Fig. 3. This is somewhat surprising since one would expect that the contribution to $\mathrm{S}$ due to para-Ps formation would correlate with I3. While that is true for typical polymers that are below the glass transition temperature [6], it has been found that for pure rubber samples, which have similar hydrocarbon composition but different morphology, $\mathrm{S}$ is essentially constant as o-Ps intensity I3 varies with the type of rubber [7]. This behavior is like that of hydrocarbon liquids, and is due to the rubber being well above its glass transition temperature. With the addition of $\mathrm{CB}$ to form the composite, part of $\mathrm{S}$ comes from annihilation of positrons in the $\mathrm{CB}$. Thus the $\mathrm{CB}$ changes the value of $S$, but the part due to the rubber remains independent of the type of rubber. The $S$ parameter thus tends to reflect mainly the properties of the $\mathrm{CB}$ in the composite.

\section{Summary}

Both o-Ps pickoff intensity, I3, and the S parameter can be useful to characterize the rubber-CB composite materials with different $\mathrm{CB}$ properties. The o-Ps pickoff lifetime does not depend on the $\mathrm{CB}$ properties. The behavior of I3 with CDBP or N2SA can be understood as due to an increase in the probability of positron annihilation within the $\mathrm{CB}$ in the composite and a corresponding reduction in positronium formation in the rubber that depends on surface area and structure or morphology of the CB particles. The $\mathrm{S}$ parameter, unlike $\mathrm{I} 3$, is independent of the type of rubber [7]. However, the part of the $S$ parameter from the positron annihilation in the $\mathrm{CB}$ depends on the surface area and structure of the $\mathrm{CB}$ particles.

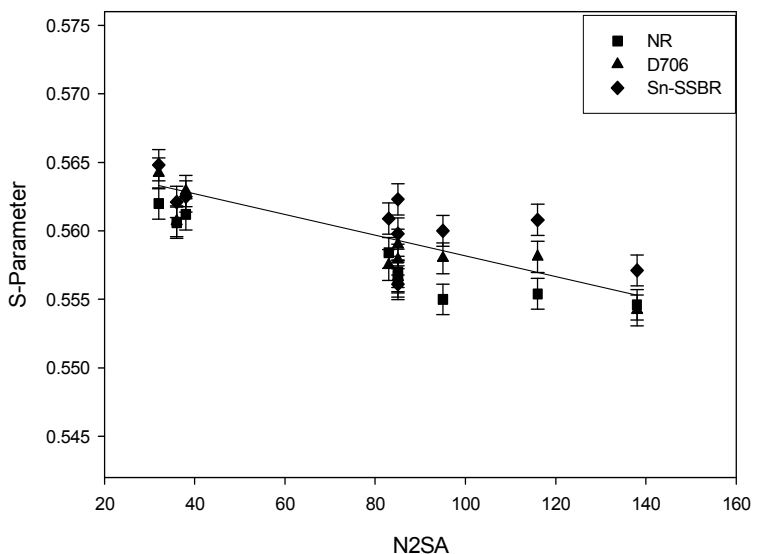

Fig. 3 The S parameter vs N2SA

\section{Reference}

[1] J. Kansy: Nucl. Instrum. Meth. Phys. Res. A374 (1996) 235.

[2] Jean-Baptiste Donnet, et al.: Carbon Black: Science and Technology, Dekker, NY (1993).

[3] M.E. Semaan, et al.: Polymer Degradation and Stability 75 (2002) 259.

[4] S. Brunauer, P. Emmett and E. Teller: J. Am. Chem. Soc. 60 (1938) 309.

[5] Y. C. Jean: Microchem J. 42 (1990) 72.

[6] K. Sato, et al.: Phys. Rev. B71 (2005) 012201.

[7] C. A. Quarles, et al.: Nucl. Instrum. Methods Phys. Res. B261 (2007) 875. 